\title{
El Desarrollo Sostenible, un pilar fundamental para la protección de los Derechos Humanos
}

Sustainable Development, a fundamental pillar

for the protection of the Human Rights

\author{
Abog. Luis Ovidio Chinchilla Fuentes* \\ Resumen
}

La historia de la sobre explotación de la tierra y de excesos y/o abusos por parte de la humanidad, tiene su origen en la Revolución Industrial. Lastimosamente, no es hasta dos siglos más tarde que hemos comenzado a percatarnos de los adversos resultados de nuestra actividad y del acelerado deterioro que ha sufrido el mundo.

A partir de este contexto, se analiza el nacimiento del concepto de Derecho al Desarrollo en los años 70's, sus principales características, así como la vinculación directa con los Derechos Humanos basada en los principios de universalidad, indivisibilidad e interdependencia.

Observando un orden lógico, se estudiarán de manera particular las responsabilidades estatales de "proteger, respetar y garantizar", y empresariales de "proteger, respetar y remediar" a la luz de los derechos humanos. Así también se analizará el apoyo que los sujetos de derecho internacional deben brindarse entre sí, con el fin de garantizar el goce de todos los derechos para todos y todas, en virtud de la solidaridad con la humanidad y de la responsabilidad internacional extraterritorial del Estado, que actualmente se encuentra en la mesa de debate.

Todo lo anterior con el objetivo de aportar planteamientos científicos para la resolución de la discusión del derecho internacional sobre las obligaciones internacionales extraterritoriales de los Estados.

Finalmente, las conclusiones respectivas del tema.

Palabras Clave: Desarrollo Sostenible, Derechos Humanos, responsabilidad internacional, responsabilidad internacional extraterritorial, desarrollo económico, empresas transnacionales.

\begin{abstract}
The history of over-exploitation of earth and the excess and/or abuses on part of humankind, has its origins in the Industrial Revolution. Unfortunately, it's not until 2 centuries later that we start to acknowledge the nefarious results of our activity and the accelerated deterioration the world has suffered.

Within that context, it's going to be analyzed how the concept of the Right to Development is born, in the 70's, the main characteristics, as well as the direct link with human rights, all based on the principles of universality, indivisibility and interdependence.

Observing a logical order, it's proceed to study particularly the states responsibilities to "protect, respect and guarantee", and company responsibilities to "protect, respect and remedy." at the light of the human rights. As well it would be analyzed the mutual aid that international law subjects must provide among them, in order to ensure the enjoyment of all rights for everyone, under the arguments of solidarity with humanity and international extraterritorial responsibility of the states which is currently at the discussion table.

All this with the objective to provide a scientific approach for the resolution of this discussion of international law about the international extraterritorial obligations of states.

Finally, the respective conclusions of this topic.
\end{abstract}

Key Words: Sustainable development, human rights, international responsibility, international extraterritorial responsibility, economic development, transnational companies.

Fecha de Recepción: martes 03 de mayo 2016

* Oficial en Derechos Humanos. Secretaria de Derechos Humanos, Justicia, Gobernación y Decentralización

Email:luisovidio45@hotmail.com

Fecha de Aprobación: lunes 31 de octubre 2016 


\section{Introducción}

Prima Facie debemos tener en cuenta diversos factores contextuales como la gran asimetría que existe entre las sociedades alrededor del mundo (países desarrollados y países en desarrollo), la brecha entre ricos y pobres ha aumentado exponencialmente en los últimos años y recordar que ya desde finales de los años 80's la Organización de las Naciones Unidas (ONU), ha impulsado la implementación del Desarrollo Sostenible (en adelante DS) a lo largo y ancho del globo terráqueo, ello a través del Informe de Brundtalnad, no obstante, a más de 20 años de este Informe la humanidad abre sus ojos al amanecer del DS.

Igualmente, es necesario mencionar que hoy por hoy son las empresas transnacionales (en adelante ETS) y los Estados son quienes dictan las pautas y parámetros para hacer realidad los sueños de Gro Harlem Brundtland, Ex Presidenta de la Comisión Mundial sobre el Medio Ambiente y el Desarrollo (1987), de asegurar que se satisfagan las necesidades del presente sin comprometer la capacidad de las futuras generaciones para satisfacer las propias. El destacado rol que juegan las ETS se debe a su gran poder económico en el mundo, a la forma en que acentúan su control en los Estados en desarrollo y a que estos últimos muchas veces carecen de mecanismos efectivos para controlar las actuaciones de las ETS. (Zubizarreta Juan y Rabasco Jesús 2013) en tanto los Estados son quienes deben establecer las leyes respecto al DS y control de las ETS bajo su jurisdicción. En ese sentido las acciones u omisiones de estos sujetos repercuten bien sea positiva o negativamente en la protección de los derechos humanos (en adelante DDHH) tal y como se discutirá ut infra.

Bajo estos contextos el presente artículo se concentra en la discusión de dos aspectos sumamente importantes para el derecho internacional, el DS y una efectiva protección de los $\mathrm{DDHH}$, en concreto, la responsabilidad internacional extraterritorial de los estados (en adelante RIE) con respecto a sus actividades y a las actividades de sus empresas fuera de su territorio, pero suscritas a su jurisdicción, así mismo la imposición de 3 obligaciones supranacionales básicas a las ETS.

Por tanto se plantean como premisas del presente artículo, que la única manera de alcanzar el DS es a través del trabajo concomitante de los Estados entre si y de las ETS con los Estados, que es necesario establecer mecanismos judiciales extraterritoriales para el control de las ETS en torno a las violaciones al DS y a los DDHH y finalmente que los Estados donde ejerzan su jurisdicción y las empresas donde realicen sus actividades tengan siempre presente el DS, ello con el asidero científico de algunos de los más importantes doctrinarios y del corpus iure nacional e internacional al respecto.

\section{Metodologia}

El artículo despliega un enfoque de tipo cualitativo, tomando como referentes básicos, la doctrina y la jurisprudencia nacional e internacional, vinculadas a los diferentes factores contextuales que han permitido e impulsado la implementación del Desarrollo Sostenible no solamente en Honduras, sino que también, a lo largo y ancho del globo terráqueo, ello a través del Informe de Brundtalnad.

Asimismo, se buscó realizar un profundo análisis y razonamiento jurídico con relación a aquellos objetivos y políticas de estado en materia de derechos humanos, los cuales deberían estar enfocados en el constante mejoramiento de la calidad de vida de todos sus habitantes.

\section{Capítulol Nacimiento y Evolucion del Desarrollo Sotenible}

Surge como primera necesidad identificar de manera precisa ¿de dónde viene el DS?, ¿dónde estamos?, ¿qué es actualmente? y ¿hacia dónde nos dirigimos en la aplicación e interpretación progresiva de esta norma?

En la década de los 70's, se realizaron los primeros análisis respecto a los efectos nocivos de la sobreexplotación de los suelos, lo que llevo a que se crearan diversos convenios para la protección del medio ambiente (Convenio de Ramsar 1971, Conferencia de Estocolmo 1972, MARPOL 73/78 y el Convención sobre el Comercio Internacional de Especies Amenazadas de Fauna y Flora Silvestres 1973 entre otros). Asimismo (Los Límites del Crecimiento 1972) menciona que el incremento de la población puede alcanzar el límite de la capacidad de alojamiento de la tierra y que nuestra depredación es la que nos está llevando a traspasar esos límites, dicha información es refrendada por la Organización Internacional Global Footprint Network, (véase http://www.footprintnetwork. org/es/index.php/GFN/page/world_footprint/ consultado el 14 de Mayo de 2016); hasta que finalmente el Informe de Brundtland de 1987 es el primer documento en utilizar el término "Desarrollo Sostenible" (Brundtland Gro Harlem 1987).

Desde ese momento la humanidad empieza a tener mayor conciencia sobre el medio en que despliega su actividad y los medios utilizados para la producción de sus bienes. En ese sentido las sociedades actuales han comprendido que el mundo no es de su propiedad y que no pueden hacer con él lo que deseen, sino que la Tierra tiene sus límites que debemos de respetar puesto que en su inmensidad, somos nosotros sus huéspedes.

El Informe de Brundtland, fue uno de los primeros documentos en preocuparse por el medio ambiente y recopilar 


\section{El Desarrollo Sostenible, un pilar fundamental para la protección de los Derechos Humanos}

las que a la fecha eran las mayores afrentas que enfrentaba el DS y que al día de hoy los países en desarrollo siguen enfrentando (1987). Pues "todos los problemas importantes relacionados con los desastres en el tercer mundo son esencialmente problemas de desarrollo no resueltos" (Cruz Roja Sueca 1985). Lo que corrobora el papel trascendental que ha y sigue jugando el DS en la efectiva protección de los $\mathrm{DDHH}$.

En la actualidad una de las definiciones más completas del DS se puede encontrar en (Alianza para el Desarrollo Sostenible de Centro América 1994) dicho cuerpo legal la define como: "Un proceso de cambio progresivo en la calidad de vida del ser humano, que lo coloca como centro y sujeto primordial del desarrollo, por medio del crecimiento económico social... que se sustenta en el equilibro biológico... garantizando la calidad de vida de las generaciones futuras." Asimismo, podemos aprovechar y definir una sociedad sostenible como: "Aquella que aporta y satisface las necesidades del presente sin comprometer la capacidad de las generaciones futuras para satisfacer sus propias necesidades." (Mayor Federico 2009).

Esto marca teóricamente en donde estamos en lo que respecta al DS y en ese orden de ideas Honduras y el mundo poseen un marco conceptual y legislativo sobre él que se ha venido trabajando y sobre el cual se debe seguir trabajando para alcanzar un DS y una efectiva protección de los DDHH, pues aún falta mucho camino por recorrer en estos dos aspectos. Lo que nos lleva al último punto de este capítulo, que es determinar bajo esta concepción teórica y la práctica actual de los Estados y ETS, hacia donde nos dirigimos en la aplicación de estos conceptos.

Cabe resaltar el trabajo realizado por la ONU, OEA y demás sujetos de derecho internacional ya que resulta sumamente importante pues hemos pasado de una economía de desgaste y sobre explotación, donde la industrialización era sinónimo de la destrucción de nuestro ambiente a un modelo económico más justo, un poco más democrático y lo mejor de todo, más sostenible. No obstante, se ha reconocido que la participación de los organismos internacionales y de los Estados no ha dado los avances esperados para la implementación del DS (Zubizarreta Juan 2009) por tanto se requiere un férreo compromiso de las ETS para implementar sin dilación el DS a nivel mundial, pues como aludimos ut supra apenas estamos presenciando la alborada del mismo, pues ha sido por esa parsimonia la humanidad ha sido víctima de graves violaciones a $\mathrm{DDHH}$.

Aunado a lo anterior, es importante destacar que las actuaciones pasadas, así como la incapacidad de los Estados en desarrollo de controlar las actividades nocivas de las ETS y la apatía de estas últimas por solucionar los daños causados a través de los años, repercuten no solo en las generaciones presentes, sino también en las futuras generaciones que hoy no tienen voz ni voto para oponerse a nuestras decisiones. De igual forma la juventud será la que viva la continua desertificación del planeta, la lluvia acida, la continua desaparición de las especies y la escasez de aire fresco, (Brundtland Gro Harlem 1987) por lo que está en nuestras manos asegurar las necesidades presentes sin comprometer a las futuras (Alfonso Dubois Migoya 2013).

En conclusión, las discusiones se están dirigiendo por un lado al establecimiento de RIE de los estados y por otro lado a la imposición de obligaciones jurídicas internacionales a las ETS. En cuanto al primer ámbito ha quedado evidenciado que la actividad de los países desarrollados afecta no solo a la circunscripción donde efectué su actividad, sino que ella tiene efectos supranacionales lo que ha quedado plenamente demostrado con el cambio climático. Esto demuestra la necesidad de establecer mecanismos jurídicos extraterritoriales para el control de las ETS, ya que si se demuestra que las actividades económicas de una ETS afectan los DDHH de las personas (Eric Engle 2009) de otra circunscripción territorial sin importar su ubicación y pudiendo llegar hasta la atribución de RIE si los estados fallan en su deber de cooperación internacional o de garantía con respecto a las violaciones al DS o a los DDHH y por otro lado se debate sobre la imposición de obligaciones jurídicas supranacionales a las ETS ya que el modus operandi de estas se aprovecha excesivamente de las deficiencias estructurales de los Estados en desarrollo y explota inescrupulosamente sus recursos y que los estados desarrollados establezcan mecanismos de control más rigurosos a las ETS que posean su casa matriz en su territorio. En ese sentido si una ETS viola DDHH en un estado huésped, las victimas de dicha violación deberán tener la posibilidad de acudir a los tribunales de los estados desarrollados bajo cuya jurisdicción se encuentre la casa matriz de dicha ETS, para reclamar sus derechos vulnerados. Esto en base al principio de solidaridad humana y cooperación internacional (Declaración del Milenio 2000).

\section{Capítulo II Responsabilidades Estatales y Empresariales}

Ahora que hemos establecido un concepto sobre DS y tomando en consideración el rumbo que parece tomar el concierto de naciones para la aplicación del mismo, es menester presentar los marcos regulatorios por los cuales se rigen estas 2 personas jurídicas. En primer lugar, hablaremos de los estados y de sus responsabilidades a partir de la creación de la ONU en 1945 y como estas se han intensificado. En segundo presentaremos el corpus iure que en la actualidad rige, aunque de manera autoexhortativa las ETS. 
Hace más de 60 años, con la creación de la Declaración Universal de los Derechos Humanos en 1948 se dio vida y exigibilidad a ciertos $\mathrm{DDHH}$, pues se establecieron por primera vez compromisos internacionales a la fecha sui generis ya que en estos documentos se consagran responsabilidades internacionales de los Estados frente a los individuos. Estas han evolucionado gracias al principio de interpretación evolutiva, el cual consagra que los instrumentos de DDHH son instrumentos vivos los cuales deben de ser interpretados de acuerdo a la evolución de los tiempos y las condiciones de vida actuales (Corte IDH 1999). Gracias a este principio y al de progresividad (Vásquez Luis y Serrano Sandra 2011), es que en la actualidad aunque nominalmente poseemos los mismos derechos que cuando se firmó este tratado, estos han logrado un alcance y efecto útil mucho mayor.

Por lo que, las obligaciones de los estados a saber, proteger, respetar y garantizar (CIDH 2015) han aumentado, pese a que nominalmente sus obligaciones internacionales siguen siendo las mismas. Ello significa que deben cumplir directamente con la norma establecida bien sea de manera positiva implementado políticas públicas adecuadas en favor de la creación de empleo, educación, política energética y DS por citar algunos ejemplos; o de manera negativa absteniéndose de violar con sus actuaciones, DDHH como el debido proceso, la propiedad privada, la libertad, la vida entre otros; y finalmente están jurídicamente obligados a organizar su aparato gubernamental con el fin de evitar que particulares y para efectos del presente artículo ETS cometan actos tendientes a vulnerar DDHH (CIDH 2015). En otras palabras, como consecuencia de la obligación de garantizar los Estados poseen 4 obligaciones especiales que son prevenir, investigar, juzgar y sancionar, procurando según el caso concreto el restablecimiento del derecho conculcado o en su caso la reparación. (Ferrer Mac-Gregor, Pelayo Carlos 2012). Ut infra se presentarán los argumentos por los cuales los Estados pueden llegar a aplicar en un futuro cercano la obligación de garantía en relación a actos cometidos por ETS con su casa matriz en su territorio, ello en base a la interpretación evolutiva y el principio de solidaridad humana llegando hasta la llamada RIE, misma que ha sido interpretada y aplicada en casos como: TEDH. Drozd y Janousek vs. Francia y España, Sentencia de 26 de junio de 1992, TEDH. Loizidou vs. Turquía. Sentencia de 23 de marzo de 1995 y TEDH. Bankovic y otros vs. Bélgica y otros. Sentencia de 12 de diciembre de 2001.

Mucho más se podría decir de las responsabilidades estatales ya que es un tema sumamente extenso y relevante. Sin embargo, es momento de pasar al análisis de las responsabilidades empresariales.

En primer lugar en 1999 entró en vigencia la "Convención para Combatir el Cohecho de Servidores Públicos Extranjeros en Transacciones Comerciales Internacionales", siendo ésta el primer intento por ejercer control extraterritorial a las ETS en materia de corrupción. Años más tarde, en 2012 se crean los "Principios Rectores Sobre Empresas y Derechos Humanos", producto de un esfuerzo de investigación y estudio por parte de la ONU para la efectiva protección de los DDHH y el DS, pues el citado organismo entendió que dadas las características de las ETS, la forma en que estas realizan sus actividades y las debilidades de muchos estados huéspedes en controlar estos gigantes financieros, existe la necesidad de extender algunas "obligaciones" internacionales a estas entidades. En el mismo año se crean los Principios Maastricht, siendo estos últimos los documentos jurídicos más importantes en cuanto a la imposición de controles internacionales de las ETS, cabe destacar que los últimos dos documentos no poseen coercibilidad jurídica internacional, es decir, que no son exigibles, sino que más bien sirven como ilustración o buenas practicas a seguir tanto para los estados pero principalmente para las ETS.

En la actualidad, estas dos entidades de derecho (Estados y ETS) tienen cierta cuota de responsabilidades frente al Derecho Internacional de los Derechos Humanos (en adelante $\mathrm{DIDH})$, responsabilidades que cada día evolucionan debido su carácter progresivo y es así que se ha llegado a crear el marco regulatorio no vinculante, supra aludido para las ETS. En tal sentido y a manera de conclusión podemos identificar que los Estados tienen la obligación de proteger, respetar y garantizar (CIDH 2015), en cambio las ETS tiene la obligación de proteger, respetar y remediar (ONU 2012). Lo que significa que en la actualidad poseen, aunque de manera volitiva "obligaciones internacionales" propias.

In fine, el impacto (positivo o negativo) que generan las acciones conjuntas de los estados y las ETS, dejaran su marca en el desarrollo de los países en desarrollo, ya que el DS como se ha concebido en el presente artículo únicamente puede realizarse con el trabajo concomitante de estos 2 agentes, toda vez que se establezcan políticas públicas y directrices estatales, así como planes de acción de las ETS que tengan como objetivo al ser humano y la obtención de lucro sin la necesidad de explotar a las personas, medio ambiente o las falencias estructurales de los Estados. El amalgamar esas circunstancias es lo que podrá trazar las líneas para que juntos como humanidad seamos capaces de mantener una economía sostenible, de servir no solo nuestros intereses sino también los de las generaciones futuras y distribuir la abundante riqueza que produce el actual modelo económico a todos los confines del mundo. No obstante, de no realizarse cambios estructurales profundos en el ordenamiento jurídico internacional, la ruta hacia el DS y la garantía del goce de los $\mathrm{DDHH}$ de futuras generaciones será sumamente difícil; por lo que en los siguientes 2 capítulos se abordaran las responsabilidades estatales y empresariales, así como la necesidad de evolucionar ciertas nociones de derecho. 


\section{El Desarrollo Sostenible, un pilar fundamental para la protección de los Derechos Humanos}

\section{Capítulo III Responsabilidades Estatales}

Frente al concierto de naciones son los Estados los primeros encaminados a proteger, respetar y garantizar los DDHH sean civiles, políticos, económicos, sociales o culturales. En tal sentido, los Estados son los principales encargados del control de las ETS, que estas entidades de derecho privado se sometan a la ley y que ajusten sus actividades al derecho al desarrollo y a la soberanía de los pueblos y naciones (Zubizarreta Juan et al. 2013). No obstante, este paradigma está cambiando debido al actual modelo económico, a la magnitud y poder que han alcanzado las ETS. Sin embargo este punto se discutirá a profundidad en el capítulo final del artículo.

Dicho lo anterior es importante tener en cuenta que: "El Estado debe reducirse en todo lo que implique control social... debe expandirse, desde la democracia participativa, a las políticas públicas relacionadas con la educación, sanidad, cultural..." (Zubizarreta Juan et al. 2013), de igual forma debemos tener presente que: "Los estados periféricos, pierden competencias, capacidad soberana por las imposiciones normativas de los Estados dominantes, Empresas Transnacionales" (Zubizarreta Juan 2009), tomando estos conceptos como un punto de partida se puede verificar la necesidad de que los Estados desarrollados apoyen a los países en desarrollo en la protección de los DDHH y la aplicación del DS, pues por una parte los estados desarrollados tienen a su alcance la posibilidad ejercer una mayor fiscalización a las ETS en el marco de sus obligaciones de proteger y garantizar (CIDH 2015) y por la otra los Estados en desarrollo requieren el apoyo de sus homólogos para lograr controlar a las ETS ya que como se mencionó estos pierden capacidad soberana frente a las ETS (Zubizarreta Juan 2009).

De acuerdo al DIDH los Estados deben asegurar el ejercicio de los DDHH a las personas dentro de su jurisdicción (Steiner Christian y Uribe Patricia 2014) y en ese sentido de acuerdo a diversas Cortes Internacionales (CIJ. 1986), (TEDH. 1992), (TPIY. 1999) y (TEDH. 2001) el termino jurisdicción no debe confundirse con el termino territorio, por lo que un estado que posea dentro de su jurisdicción la sede de una ETS deviene en la obligación internacional de asegurarse que las actividades que realice esta empresa dentro de su jurisdicción estén enmarcadas en el DS y no vulneren DDHH, pues de no ser así el Estado podría enfrentar responsabilidad internacional, ya que esta obligación se enmarca dentro de la obligación general de garantía supra referida. Empero existen múltiples ejemplos en los que las actividades de las ETS han afectado el DS de individuos que se encuentran fuera de las fronteras teniendo como un común denominador que estas afectaciones se producen en Estados en desarrollo, como el del sindicato Sinaltrainal contra Nestlé al envasar leche caducada en Colombia, el Caso Comunidades de Parej Oriental vs. Coal India Ltda., el uso de Nemagon por empresas como Shell, Dole y Chiquita en Honduras y Nicaragua y demás infra aludidos.

Pese a las dificultades fácticas que existen en el control de las ETS los Estados siguen siendo los encargados de someter al imperio del derecho a las mismas. Para tales efectos en la actualidad los Estados en desarrollo, generalmente estados huéspedes, necesitan la ayuda de los estados desarrollados, es decir el apoyo de los estados cuyos ordenamientos jurídicos son más sólidos y en donde las empresas tienen generalmente sus Casas Matrices. Esto es así en virtud de que las ETS aprovechan la debilidad de los ordenamientos jurídicos de los Estados huéspedes (Özden Melik y Teitelbaum Alejandro 2011) y se mueven en un marco normativo muy favorable como los tratados de comercio y regímenes jurídicos especiales (CIDH 2015) como describiremos en el último capítulo.

Un claro ejemplo de los postulados que hemos mencionado a lo largo del presente artículo y del control de las actuaciones de las ETS fuera de su jurisdicción por parte de los Estados desarrollados, particularmente de Estados Unidos es el Alien Tort Claims Act (en adelante ATCA), aunque esta ley limita significativamente el rango de protección de los DDHH ya que no protege derechos económicos, sociales y culturales, menos aún el Derecho al Desarrollo, permite la posibilidad de demandar a una empresa con sede en los Estados Unidos por las actividades que realice fuera del territorio siempre y cuando estas acciones laceren $\mathrm{DDHH}$ y que dichas actuaciones hayan sido planificadas o se tuviera el conocimiento suficiente por parte de la casa matriz (Zamora Francisco 2012).

Aunque este mecanismo resulta efectivo en algunas ocasiones presenta ciertos inconvenientes. Para mencionar algunos ejemplos: ¿El conocimiento que puedan tener los profesionales de otras latitudes de este mecanismo legal y los costos que representa hacer uso del ATCA?, ¿Cómo las personas de escasos recursos, indígenas o niños explotados de países en desarrollo puedan acceder a este mecanismo de protección? y ¿Dónde y cómo ejecutar una sentencia que dictada en los Estados Unidos condene a un empresa que funciona en Suramérica? (Vieira Fernando y Pinheiro Thiago 2010). Otro mecanismo de protección de los Estados es el Companies Act del Reino Unido, sumamente similar al ATCA.

Como podemos apreciar algunos Estados ya han adoptado políticas públicas y han comenzado a organizar su aparato gubernamental, realizando actuaciones en el marco legislativo y judicial no solo de protección a sus nacionales sino a cualquier ser humano, incluso aquellos que se encuentran a miles de kilómetros de sus fronteras. Asimismo los organismos internacionales de protección de los DDHH deben mantenerse a la vanguardia en la interpretación del corpus 
iure de $\mathrm{DDHH}$, como actualmente lo está realizando la Corte Interamericana de Derechos Humanos al admitir la solicitud de opinión consultiva presentada por Colombia, relativa a al impacto de grandes proyectos en el medio ambiente marino, específicamente en la región del Gran Caribe, (http://www. corteidh.or.cr/cf/Jurisprudencia2/solicitud_opiniones_consultivas.cfm?lang=es consultado el 18 de Junio de 2016) y finalmente el apoyo a los Estados en la creación e implementación de mecanismos de control tendientes a asegurar el ejercicio de todos los DDHH y el DS.

Si bien es cierto que los Estados en desarrollo poseen las mismas responsabilidades que los estados desarrollados, no es menos cierto que su capacidad para cumplir con estas obligaciones es exponencialmente menor. Al igual que sus homólogos deben fiscalizar a las ETS que realicen operaciones dentro de su jurisdicción, lo cual resulta complicado cuando se pone en contraste el poder de algunas ETS frente al de un país como Honduras donde según datos oficiales del INE el $68.7 \%$ de la población vive en la pobreza y el $44.7 \%$ vive en pobreza extrema(http://www.ine.gob.hn/index.php/ component/content/article?id=91 consultado el 4 de Junio de 2016) y cuyo PIB es de 19.39 millones de dólares (http:// www.bancomundial.org/es/country/honduras consultado el 4 de Junio de 2016) en comparación con el de algunas de las empresas más grandes como Apple que en 2015 generó 53.7 billones de dólares en ganancias, Wal-Mart que solo en el año 2015 sus ventas ascendieron a 482.1 billones de dólares o incluso Nestlé cuyo valor de mercado es 12,155.75 veces más alto que el PIB de Honduras (http://www.forbes.com/global2000/list/\#header:marketValue_sortreverse:true consultado el 4 de junio de 2016). Otro aspecto que se debe tener muy presente en esta ecuación es la competencia que existe entre los países en desarrollo por atraer la inversión de estos grandes capitales mediante la creación de paraísos fiscales, regímenes especiales de trabajo, otorgando concesiones y cuya seguridad jurídica es casi inexistente, son solo algunos de los motivos de porque los países del África subsahariana, América central o del caribe son explotados por las ETS. Por lo que en atención al principio de solidaridad humana y obligaciones del principio de beneficio recíproco y con equidad global (Alfonso Dubois Migoya 2013) se requiere establecer a los estados desarrollados la obligación de aportar al control extraterritorial de sus ETS, lo cual es posible a través de la obligación general de garantizar y la obligación especifica de prevenir materializada a través de las acciones de fiscalizar (RED- DESC 2014).

In fine las principales responsabilidades de los estados en torno al DS, DDHH y ETS son por un lado la creación de leyes que aseguren una economía de sostenible, el pleno ejercicio de los DDHH, ser capaces de sujetar a las ETS a las leyes de su país, haciendo una mención especial en cuanto a los Estados desarrollados que deben de establecer mecanismos de protección extraterritorial con base al principio de solidaridad humana y final, y paulatinamente mejorar las condiciones de vida de las personas a través de un DS y cooperación internacional. Aunque en la práctica es más difícil de lo que podría parecer.

\section{Capítulo IV Responsabilidades Empresariales}

Aunque poseamos una noción clara de lo que es una ETS ahora necesitamos definir con precisión científica que es. $Y$ para tales efectos haremos uso de la definición de (Özden Melik et al. 2011) la cual establece: "se refiere a una entidad económica o a un grupo de entidades económicas que operan en dos o más países, sin importar su marco jurídico, el país de origen o el de establecimiento, o que su acción sea individual o colectiva. Las sociedades transnacionales son personas jurídicas de derecho privado con una implantación territorial múltiple, pero con un centro único para las decisiones estratégicas."

Establecida la definición, es nuestro menester analizar las responsabilidades de las ETS, que en la actualidad deben ir más allá de simples códigos de conducta, pues las prácticas pasadas y actuales nos hacen ver la imperiosa necesidad de que ellas se sujeten a más que un código de conducta, ya que de esta forma deben asumirse de manera autoexhortativa las obligaciones de proteger, respetar y remediar supra mencionadas (Zubizarreta Juan 2009). Igualmente deben trabajar concomitantemente con los Estados para que todos los sujetos de la humanidad puedan ejercer plenamente sus $\mathrm{DDHH}$, ya que a través de sus actividades se puede favorecer o afectar una gran cantidad de DDHH como el Derecho al Desarrollo. Para los efectos del presente artículo, se analizará con un énfasis particular del DS, siendo algunos aspectos principales los daños ambientales, los derechos de los pueblos, el principio de precaución y las negligencias graves que han dado lugar a la muerte a miles de personas (Ozden Melik et al. 2011).

En cuanto a las obligaciones de las ETS debemos entender que los términos proteger y respetar se entenderán bajo el criterio mutatis mutandis, lo que quiere decir que las ETS deben realizar actuaciones en pro de los DDHH y del DS, como el respetar el pago de los salarios mínimos y las jornadas laborales (Albert Sales I Campos 2013), poseer planes de contingencia, respetar los límites de explotación de los recursos naturales impuestos por los estados y cumplir con los estudios de impacto ambiental, entre otros. En conclusión, se deben prevenir las actuaciones que laceran el DS o los $\mathrm{DDHH}$, es decir, se deben de realizar acciones positivas bajo el marco jurídico estatal y abstenerse de infringir los $\mathrm{DDHH}$ y al DS (ONU 2012). 


\section{El Desarrollo Sostenible, un pilar fundamental para la protección de los Derechos Humanos}

No obstante la obligación de remediar representa un criterio novedoso pues significa que las ETS deben mejorar el acceso a las vías de reparación de las víctimas de sus actuaciones. Dichos mecanismos deben seguir ciertos parámetros establecidos en el Principio Rector 31 de los "Principios Rectores sobre Empresas y Derechos Humanos", como ser la legitimidad, accesibilidad, predictibilidad, equidad y transparencia, mismos que deben ajustarse a las costumbres y medios usuales de reclamación en las regiones que desarrollen su actividad, ello con el fin de asegurar su eficacia.

Por lo que podríamos concluir que las ETS deben ajustarse a las leyes de cada país y no deberán valerse de las debilidades de algunos Estados para obtener un provecho económico que incluso pueda ser insultante a la pobreza que enfrenta la humanidad (Özden Melik et al. 2011). En virtud que en este momento "las sociedades transnacionales acentúan su control sobre los recursos naturales del planeta, dictan su voluntad a los Estados más débiles y explotan a las poblaciones." (Zubizarreta Juan 2009).

Siguiendo la misma rubrica existen numerosos ejemplos de las nefastas violaciones a DDHH que perpetran las ETS a lo largo y ancho de nuestro planeta, casos como los ut supra aludidos y otros como el Caso Wiwa vs. Royal Dutch Petroleum Company y Shell Transport Ltda., el Caso Comunidad Dongria Kondh vs. Vendata Alumina Ltda., el caso de Novartis en India y la catástrofe de Bhopal India (http://www.bhopal. com/ consultado el 25 de junio de 2016), como una de las peores de la historia de la humanidad entre otras.

Por tales motivos fue necesario encuadrar las actividades de las empresas bajo los parámetros ut supra aludidos, en virtud de que las empresas son las personas de derecho privado que puede ayudar al desarrollo de un pueblo y en tal sentido también tienen la responsabilidad de facilitar el camino a la globalización y al DS (Brundtland Gro Harlem 1987), así cuando desarrollen sus actividades deben de tener en cuenta los problemas ambientales que puedan causar sus acciones (Brundtland Gro Harlem 1987) y en su caso las medidas de reparación necesarias cuando la mitigación y prevención no hayan sido suficientes para prevenir actos graves que limiten el pleno ejercicio de los DDHH (Zubizarreta Juan 2009).

Enfoquémonos ahora en el escenario anterior a un hecho que lesione DDHH o el DS y en tal contexto las ETS deben realizar todo lo necesario para evitar que una posible violación sea cometida, que según los estándares internacionales seria la realización de todas las Evaluaciones de Impacto Ambiental, la consulta previa libre e informada, (DPLF OXFAM 2011) obtener el permiso de operaciones sobre el rubro respectivo. Es trascendental mencionar que las evaluaciones deben ser realizadas en base a criterios objetivos, por profesionales competentes y sin utilizar ninguna práctica poco ética o que se configure como competencia desleal (Zubizarreta Juan 2009), igualmente debe estar de acuerdo a los Principios de Derecho Internacional del Medio Ambiente, como ser el principio de buena vecindad, principio de acción preventiva, principio de precaución, obligación de indemnizar por daños y por último pero no menos importante el principio del desarrollo sostenible (Soto Max) y al DIDH.

Si los Estados han logrado sujetar a las ETS a su legislación y han cumplido con los parámetros internacionales para operar y a pesar de esto sucede alguna catástrofe o accidente, estaremos en ese escenario frente a las obligaciones de las ETS ex post facto y bajo estos términos debemos tener muy en cuenta los "Principios Rectores sobre Empresas y Derechos Humanos" en donde se fijan directrices prácticas y funcionales para remediar las violaciones que se cometan en el ejercicio de su actividad, como procedimientos internos de reclamación (ONU 2012) y la justa indemnización de las victimas toda vez que una respuesta tardía puede dar lugar a una situación irremediable y a una posible condena internacional.

Definitivamente las ETS tienen una gran responsabilidad frente a los DDHH y de acuerdo a las tendencias en el derecho internacional esta responsabilidad será cada vez mayor, así toda vez que no cumplan con las responsabilidades que le acarree tanto el Derecho al Desarrollo, los DDHH y finalmente pero no de menor importancia el derecho interno pueden influir de manera nefasta en el desarrollo de todo un país. En tal sentido, las ETS con su gran poder económico devienen en la gran responsabilidad de ayudar a las naciones a desarrollarse de manera equitativa en el mundo.

\section{Conclusiones}

- El Derecho al Desarrollo y el DS requieren un mayor empuje y una mayor socialización, quizá sean la mejor solución para que los países salgan del subdesarrollo.

- El enfoque global de la economía debe cambiar y adecuarse no solo a obtener el máximo de lucro en el menor tiempo posible como sucedía en el pasado, sino que ese paradigma debe ser modificado para enfocarse en la sostenibilidad de la economía y la capacidad de que las generaciones venideras disfruten de un ambiente sano.

- El desarrollo duradero requiere una visión de las necesidades futuras de los humanos incorporando variables como la educación, el medio ambiente y la salud.

- $\quad$ El gran problema de la desigualdad entre las naciones es la desigualdad del acceso en los recursos y en este ámbito las ETS deben de jugar un rol preponde- 
rante en promover el desarrollo y no solo la obtención de lucro a costas de los estados en desarrollo.

- Los objetivos del Estado deben ser enfocados en el constante mejoramiento de la calidad de vida de sus habitantes y por lo tanto no deben aceptar ninguna condición que sea contraria a estas condiciones. De igual forma deben adecuar su aparato gubernamental para controlar a las ETS, incluso en el caso de los estados desarrollados en relación a las ETS que operen fuera de sus fronteras.

- Todos los Estados del mundo, en especial los desarrollados tienen el compromiso de solidaridad con toda la humanidad para hacer un bloque que no solo beneficie sus intereses, sino que sea lo mejor para la humanidad y erradicar la pobreza extrema, el analfabetismo y propiciar el uso sostenible de los recursos.

- La pobreza es uno de los factores que no solo limita el crecimiento económico y que per se representa una limitación en el ejercicio de los $\mathrm{DDHH}$, sino que también consume y degrada el ambiente y afecta el DS.

- Los DDHH y el DS no son enemigos de la economía 0 de la inversión, sino que deben verse como el camino de una sociedad democráticamente justa y sostenible hacia el progreso.

- La comunidad internacional debe emprender los esfuerzos necesarios para discutir la RIE de los Estados y las obligaciones internacionales de las ETS.

- Las ETS deben de regirse por un cuerpo jurídico internacional coercitivo.

- El desarrollo económico no debe sobreponerse al desarrollo social y menos aún al mejoramiento de la calidad de vida de las personas, sino que se deben de adoptar políticas tanto endógenas como exógenas que busquen a través de los beneficios que ofrece el libre mercado la protección a los DDHH y al DS.

- Antes de iniciar procesos de explotación se requiere la consulta previa, libre e informada de los habitantes de la zona que se desea explotar.

- Las ETS no deben permitir la realización de actividades que, por ellas, sus filiales o sus distribuidores puedan violar DDHH o afectar negativamente el desarrollo de los pueblos.

- Cuando existan violaciones a DDHH las ETS deben remediar de manera proporcional y mediante el principio del Restitutium In Integrum.

\section{Agradecimientos}

Quisiera agradecer a la Universidad Nacional Autónoma de Honduras y al Instituto de Investigación Jurídica de la Facultad de Ciencias Jurídicas, por considerar este artículo para publicarlo en la Revista de Derecho, a la abogada Gina Larissa Reyes Vásquez, quien proporcionó su apoyo en la revisión y redacción de este árticulo. Y por último, pero no menos importante, al Programa de Juicios Simulados en materia de Derechos Humanos Moot Court UNAH que sembró en mí el espíritu de investigación y la convicción de un mundo mejor a través del respeto a los Derechos Humanos.

\section{Bibliografía}

- Cruz Roja Sueca (1985) Más vale prevenir que curar; Informe sobre desastres que afectan al hombre y al medio ambiente en el tercer mundo, pág. 177, Estocolmo Suecia.

- Brundtland Gro Harlem (1987) Informe de la Comisión Mundial sobre el Medio Ambiente y el Desarrollo, párr. 7,12, 14, 16, 20, 25, 27, 68, 80, 103, Cap. 1 párr. 27, 42, Cap. 2 párr. 55,65 , Cap. 3 párr. 58,60 , Cap. 5 párr. 82, Cap. 8 párr. 92.

- ONU, (2000). Declaración del Milenio, párr. 4,6, 9, 23, 26 y 30.

- ONU, (2012) La Responsabilidad de las Empresas de Respetar los Derechos Humanos Guía para la Interpretación, pág. 1-3, 9, 16.

- ONU, (2012) Principios Rectores sobre Empresas y Derechos Humanos, Principio 29 y 31.

- $\quad \mathrm{CIDH},(2015)$. Pueblos indígenas, comunidades afrodescendientes y recursos naturales: protección de derechos humanos en el contexto de actividades de extracción, explotación y desarrollo párrs. 6, 14, 15, 20, 39 y 40 Washington. D.C, OEA.

- Alianza para el Desarrollo Sostenible de Centro América (1994) Concepto de Desarrollo Sostenible, Managua, Nicaragua.

- Mayor Federico (diciembre 2009) Los Límites del Crecimiento, Fundación Dialnet Universidad de la Rioja VOL. 181, pág.14. 


\section{Legislación con enfoque de Derechos Humanos para la gestión de Desarrollo Humano Sostenible en Honduras}

- Zubizarreta Juan (2009) Las Empresas Transnacionales frente a los Derechos Humanos: Historia de una Asimetría Normativa, Bilbao, Hegoa, pág. 2, 32, 83, 98, 113-119, 174, 192, 248, 249, 254.

- Eric Engle, (2009). Third Party Effect of Fundamental Rights (Drittwirkung) en Hanse Law Review Vol. 5 No. 2 Berlin, Alemania págs. 165-168.

- Vieira Fernando y Pinheiro Thiago (2010) Empresas Transnacionales en el Banco de los Acusados, Curitiba, Terra de Direitos pág. 28.

- Vásquez Luis, Serrano Sandra, (2011) Los principios de universalidad, interdependencia, indivisibilidad y progresividad. Apuntes para su aplicación práctica en Carbonell Miguel, Salazar Pedro, La Reforma Constitucional De Derechos Humanos: Un Nuevo Paradigma págs. 159-165 Ciudad de México, Instituto de Investigaciones Jurídicas.

- Özden Melik, Teitelbaum Alejandro, (2011) Sociedades Transnacionales y Derechos Humanos, Ginebra, CETIM pág. 2, 3, 7, 8, 20, 36 y 38.

- DPLF OXFAM (2011) El derecho a la consulta previa, libre e informada de los pueblos indígenas La situación de Bolivia, Colombia, Ecuador y Perú. Washington DC. DPLF OXFAM pág. 23-27.

- Ferrer Mac-Gregor, Pelayo Carlos, (2012) La obligación de "respetar" y "garantizar" los derechos humanos a la luz de la jurisprudencia de la Corte Interamericana. Análisis del artículo $1^{\circ}$ del pacto de San José como fuente convencional del derecho procesal constitucional mexicano Estudios Constitucionales Vol. 10 núm. 2 pág. 151-155.

- Zamora Francisco, (2012) La Responsabilidad de las Empresas Multinacionales por Violaciones de los Derechos Humanos: Practica Reciente, España Huriage pág. 5 y 12.

- Alfonso Dubois Migoya. (2013). Tiempo de debate: un nuevo desarrollo para nuevos tiempos, Zubizarreta Juan y Rabasco Jesús. (2013), Las Empresas Transnacionales y los Derechos Humanos y Albert Sales I Campos. (2013) Trabajo, relaciones laborales y consumo: de la "economía real" a la "economía especulativa" en Gonzalo de Castro y Begoña Román, Cambio Social y Cooperación en el Siglo XXI Vol. 2, págs. 34, 113-128 y 135-137 Barcelona: Fundación Educo.

- Steiner Christian, Uribe Patricia (2014) Convención Americana sobre Derechos Humanos comentada Mé- xico, Suprema Corte de Justicia de la Nación, Fundación Konrad Adenauer, pág. 60-61.

- RED-DESC, (2014). Economía Global, Derechos Globales guía para interpretar las obligaciones relacionadas con los derechos humanos en la economía global, Párr. 22-25, Nueva York, Estados Unidos.

- Soto Max, Principios Generales de Derecho Internacional del Medio Ambiente, recuperado de: http:// www.oas.org/dsd/Tool-kit/Documentosspa/Moduloll/ Soto\%20Article.pdf.

- http://www.footprintnetwork.org/es/index.php/GFN/ page/world_footprint/.

- http://www.corteidh.or.cr/cf/Jurisprudencia2/solicitud_ opiniones_consultivas.cfm?lang=es

- http://www.ine.gob.hn/index.php/component/content/ article?id=91

- $\quad$ http://www.bancomundial.org/es/country/honduras.

- http://www.forbes.com/global2000/ list/\#header:marketValue_sortreverse:true.

- $\quad$ http://www.bhopal.com/.

- CIJ. Nicaragua vs. Estados Unidos de América Meritos, 27 de junio 1986 párr. 110, 114 y 115.

- TEDH. Drozd y Janousek vs. Francia y España, Sentencia de 26 de junio de 1992, párr. 91.

- TEDH. Bankovic y otros vs. Bélgica y otros. Sentencia de 12 de diciembre de 2001 párr. 59-61.

- TPIY. The Prosecutor vs. Dusko Tadic 15 de Julio 1999 párr. 69 y 106.

- Corte IDH. (1999) Opinión Consultiva OC-16/99, párr. 114 San José, Corte IDH. 